\title{
Sur la rectification des lignes de courbure d'un ellipsoïde
}

(par Mr. Michael Roberts, à Dublin.)

$D_{\text {ana }}$ á la comparaison des arcs des lignes de courbure d'un ellipsoïde, qui proviennent de l'intersection de cette surface avec un hyperboloïde gauche. Dans ce qui suit je me propose de présenter la solution complète du problème, eu cherchant les formules dont dépend la comparaison des arcs des lignes de courbure, qui se trouvent situées sur un hyperboloïde à deux nappes.

Considérons maintenant le système des trois surfaces homofocales

$$
\begin{aligned}
& \frac{x^{2}}{a^{2}}+\frac{y^{2}}{a^{2}-b^{2}}+\frac{z^{2}}{a^{2}-c^{2}}=1 \\
& \frac{x^{2}}{\mu^{2}}+\frac{y^{2}}{\mu^{2}-b^{2}}-\frac{z^{2}}{c^{2}-\mu^{2}}=1 \\
& \frac{x^{2}}{v^{2}}-\frac{y^{2}}{b^{2}-v^{2}}-\frac{z^{2}}{c^{2}-v^{2}}=1
\end{aligned}
$$

oủ l'on suppose que $a>\sqrt{a^{2}-b^{2}}>\sqrt{a^{2}-c^{2}}$. On sait que l'élément de l'arc de la courbe d'intersection des surfaces (I) et (III) a pour expression $\sqrt{\frac{\left(a^{2}-\mu^{2}\right)\left(\mu^{2}-v^{2}\right)}{\left(\mu^{2}-b^{2}\right)\left(c^{2}-{ }^{2} \mu\right)}} d \mu$, et si l'on transforme cette valeur en posant

$$
\xi^{2}=\frac{c^{2}-b^{2}}{a^{2}-b^{2}}, \quad \xi^{\prime 2}=\frac{a^{2} \xi^{2}}{c^{2}}, \quad \xi^{\prime 2}=\frac{\left(a^{2}-v^{2}\right) \xi^{2}}{c^{2}-v^{2}}, \quad \mu^{2}=\frac{c^{2}-a^{2} \xi^{2} \sin ^{2} \varphi}{1-\xi^{2} \sin ^{2} \varphi},
$$

on trouve, en désignant par $s_{\varphi}$ l'arc qui dépend d'une amplitude $\phi$, compté Annali di Matematica, tomo V. 
du sommet pour lequel $\mu=c$, et en écrivant

$$
\begin{gathered}
L(\phi)=\int_{0}^{\varphi} \frac{d \varphi}{\sqrt{\left(1-\xi^{2} \sin ^{2} \varphi\right)\left(1-\xi^{\prime 2} \sin ^{2} \varphi\right)\left(1-\xi^{1 / 2} \sin ^{2} \varphi\right)}}, \\
P\left(-\xi^{2}, \phi\right)=\int_{0}^{\varphi} \frac{d \varphi}{\left(1-\xi^{2} \sin ^{2} \varphi\right) \sqrt{\left(1-\xi^{2} \sin ^{2} \varphi\right)\left(1-\xi^{\prime 2} \sin ^{2} \varphi\right)\left(1-\xi^{\prime 2} \sin ^{2} \varphi\right)}},
\end{gathered}
$$

l'expression

$$
s_{\varphi}=\frac{l a^{2}-c^{2}}{c \sqrt{\left(a^{2}-b^{2}\right)\left(c^{2}-v^{2}\right)}}\left\{\left(a^{2}-c^{2}\right) P\left(-\xi^{2}, \phi\right)-\left(a^{2}-\nu^{2}\right) L(\phi)\right\} .
$$

Maintenant en supposant que les angles $\phi, \psi, \chi, \sigma, \tau$ soient liés par les équations

$$
\begin{aligned}
& \cos \phi \cos \psi=\cos \sigma \cos \tau \cos \chi+\sin \sigma \sin \tau \sin \chi \nabla(\phi, \psi), \\
& \cos \phi \cos \chi=\cos \sigma \cos \tau \cos \psi+\sin \sigma \sin \tau \sin \psi \nabla(\phi, \chi),
\end{aligned}
$$

.où $\nabla(\phi, \psi)=$

$$
\frac{\sin \varphi \cos \varphi \sqrt{\left(1-\xi^{2} \sin ^{2} \psi\right)\left(1-\xi^{\prime 2} \sin ^{2} \psi\right)\left(1-\xi^{\prime \prime 2} \sin ^{2} \psi\right)}-\sin \psi \cos \psi \sqrt{\left(1-\xi^{2} \sin ^{2} \varphi\right)\left(1-\xi^{\prime 2} \sin ^{2} \varphi\right)\left(1-\xi^{\prime \prime 2} \sin ^{2} \varphi\right)}}{\sin ^{2} \varphi-\sin ^{2} \psi},
$$

j'ai démontré (voir ce Journal, t. $3^{\mathrm{e}}$, page 76) qu'on a

$$
\begin{aligned}
P\left(-\xi^{2}, \phi\right)+P\left(-\xi^{2}, \psi\right)+P\left(-\xi^{2}, \chi\right)-P\left(-\xi^{2}, \sigma\right)-P\left(-\xi^{2}, \tau\right)= \\
\frac{\xi^{4} \sin \varphi \sin \psi \sin \chi \sin \sigma \sin \tau}{\sqrt{\left(1-\xi^{2} \sin ^{2} \varphi\right)\left(1-\xi^{2} \sin ^{2} \psi\right)\left(1-\xi^{2} \sin ^{2} \chi\right)\left(1-\xi^{2} \sin ^{2} \sigma\right)\left(1-\xi^{2} \sin ^{2} \tau\right)}},
\end{aligned}
$$

en sorte que, si $s_{\varphi}, s_{\psi}, s_{\chi}, s_{\sigma}, s_{\tau}$ sont des ares de la ligne de courbure dont il s'agit, on obtient

$$
s_{\varphi}+s_{\psi}+s_{\chi}-s_{\sigma}-s_{\tau}=
$$

$\frac{\left(a^{2}-c^{2}\right)^{2}}{c \sqrt{\left(a^{2}-b^{2}\right)\left(c^{2}-v^{2}\right)}} \cdot \frac{\xi^{4} \sin \varphi \sin \psi \sin \chi \sin \sigma \sin \tau}{\sqrt{\left(1-\xi^{2} \sin ^{2} \varphi\right)\left(1-\xi^{2} \sin ^{2} \psi\right)\left(1-\sin ^{2} \sin ^{3} \chi\right)\left(1-\xi^{2} \sin ^{2} \sigma\right)\left(1-\xi^{2} \sin ^{2} \tau\right)}} \cdot$

Il est bon de remarquer que $\xi, \xi^{\prime}, \xi^{\prime \prime}$ sont inférieures a l'unité et qu'on a $\xi^{\prime \prime}>\xi^{\prime}>\xi$.

Maintenant soient $z_{1}, z_{2}, z_{3}, z_{4}, z_{5}$ les coordonnées dirigées suivant l'axe des $z$ des extremités variables des $\operatorname{arcs} s_{\varphi}, s_{\psi}, s_{\chi}, s_{\sigma}, s_{\tau}$, on a

$$
\frac{\sin \varphi}{\sqrt{1-\xi_{3}^{2} \sin ^{2} \varphi}}=\frac{c \sqrt{a^{2}-b^{2}}}{\left(a^{2}-c^{2}\right) \sqrt{c^{2}-v^{2}}} z_{1}
$$


et ainsi pour les autres; d'où nous tirons

$$
s_{\varphi}+s_{\psi}+s_{\chi}-s_{\sigma}-s_{\tau}=\frac{c^{4}\left(c^{2}-b^{3}\right)^{2}}{\left\{( a ^ { 2 } - c ^ { 3 } ) \left(c^{2}-\gamma^{2} \ell^{3}\right.\right.} z_{1} z_{2} z_{3} z_{4} z_{6^{\circ}}
$$

Dans l'expression que j'ai donnée pour la rectification des lignes de courbure de l'ellipsoïde (I) (voir ce Journal, t. $2^{\mathrm{e}}$, page 15) qui sont situées sur l'hyperboloïde gauche (II), les arcs sont comptés du sommet pour lequel $\nu=0$. Si l'on veut obtenir l'expression des arcs de cette courbe qui sont comptés du sommet pour lequel $\nu=b$, il faut mettre dans l'expression

$$
\sqrt{\frac{\left(a^{2}-v^{2}\right)\left(\mu^{2}-v^{2}\right)}{\left(b^{3}-v^{2}\right)\left(c^{2}-v^{2}\right)}} d \nu, \nu=\frac{b \mu \cos \varphi}{\sqrt{\mu^{2}-b^{2} \sin ^{2} \varphi}}:
$$

en sorte que $\phi$ s'annule quand $\nu=b$. Si $s_{\varphi}$ désigne l'arc dont il s'agit, qui dépend d'une amplitude $\phi$, en mettant

et en posant

$$
\eta^{\prime \prime}=\frac{b}{\mu}, \eta^{\prime}=\sqrt{\frac{a^{3}-\mu^{2}}{a^{2}-b^{2}}} \eta^{\prime \prime}, \eta=\sqrt{\frac{c^{2}-\mu^{2}}{c^{2}-b^{2}}} \eta^{\prime \prime}
$$

$$
\begin{gathered}
L(\phi)=\int_{0}^{\varphi} \frac{d \varphi}{\sqrt{\left(1-n^{2} \sin ^{2} \varphi\right)\left(1-n^{\prime 2} \sin ^{2} \varphi\right)\left(1-n^{\prime \prime 2} \sin ^{2} \varphi\right)}}, \\
P\left(-\eta^{\prime \prime 2}, \phi\right)=\int_{0}^{\varphi} \frac{1}{1-\eta_{1}^{\prime \prime \prime} \sin ^{2} \varphi} \frac{d \varphi}{\sqrt{\left(1-n^{2} \sin ^{2} \varphi\right)\left(1-n^{\prime 2} \sin ^{2} \varphi\right)\left(1-n^{\prime \prime 2} \sin ^{2} \varphi\right)}}
\end{gathered}
$$

nous trouvons

$$
s_{\varphi}=\frac{\mu^{2}-b^{2}}{\mu \sqrt{\left(a^{2}-b^{2}\right)\left(c^{3}-b\right)}}\left\{\left(a^{2}-\mu^{2}\right) L(\phi)+\left(\mu^{2}-b^{2}\right) P\left(-\eta^{\prime / 2}, \phi\right)\right\} .
$$

En suivant la marche que nous venons d'indiquer, on trouve que les arcs correspondants aux angles $\phi, \psi, \chi, \sigma, \tau$ sont liés par l'équation

$$
s_{\varphi}+s_{\psi}+s_{\chi}-s_{\sigma}-s_{\tau}=
$$

$\frac{\left(\mu^{2}-b^{9}\right)^{2}}{\mu \cdot \sqrt{\left(a^{2}-b\right)\left(c^{2}-b^{0}\right)}} \frac{n^{\prime \prime \prime} \sin \varphi \sin \psi \sin \chi \sin \sigma \sin \tau}{\sqrt{\left(1-n^{\prime \prime 2} \sin ^{2} \varphi\right)\left(1-n^{\prime \prime 2} \sin ^{2} \psi\right)\left(1-\eta_{1}^{\prime \prime 2} \sin ^{2} \chi\right)\left(1-\eta^{\prime \prime 2} \sin ^{2} \sigma\right)\left(1-n^{\prime \prime 2} \sin ^{2} \tau\right)}}$.

Si $y_{1}, y_{2}, y_{3}, y_{4}, y_{5}$ sont les coordonnées dirigées suivant l'axe des $y$ des extremités variables des arcs qui figurent dans le premier membre de cette dernière équation, le second membre s'écrit de la manière suivante

$$
\frac{b^{4}\left(c^{2}-b^{2}\right)^{2}}{\left\{\left(a^{2}-b^{2}\right)\left(\mu^{2}-b^{2}\right)\right\}^{3}} y_{1} y_{2} y_{3} y_{4} y_{4}
$$

Collège de la Trinité à Dublin, mars 1871. 